\title{
Prevalence of voice \& swallowing complaints in Pre-operative thyroidectomy patients: a prospective cohort study
}

Theresa Holler ${ }^{1}$ and Jennifer Anderson ${ }^{1,2^{*}}$

\begin{abstract}
Background: Compressive symptoms are common in patients with thyroid disease and are an accepted indication for thyroidectomy. The objective of this study was to evaluate the prevalence and severity of voice and swallowing complaints in pre-operative thyroidectomy patients and to compare these with thyroid volume, thyroid pathology and laryngopharyngeal reflux.
\end{abstract}

Methods: A consecutive case series design was performed. All patients undergoing a thyroidectomy (partial or total) at a tertiary care centre during a 2 year period were invited to participate. Fifty nine (10 male, 49 female) aged 19-73 were enrolled (59.3\% total thyroidectomy, 40.7\% partial thyroidectomy). Voice and swallowing complaints were subjectively evaluated using the Voice Handicap Index (VHI) and the Modified Swallowing Impairment Score (MSIS), respectively. All patients completed the Reflux Symptom Index (RSI) and underwent videostroboscopy. Vocal function was objectively evaluated using perceptual ratings (GRBAS) and acoustic voice analysis (jitter, shimmer, maximum phonation time, maximum fundamental frequency range). The relationship between voice and swallowing symptoms was compared with thyroid volume and surgical pathology.

Results and discussion: The average VHI score was 8.39, representing normal voice scores. Of the objective voice measures, only jitter and a subset of GRBAS measures were slightly elevated. Swallowing complaints were reported at least "some of the time" by 25 patients (41.7\%), and "often or always" by 16 patients (26.7\%). Of the patients with voice and swallowing complaints, $68.4 \%$ and $56 \%$, respectively, had elevated RSI scores consistent with laryngopharyngeal reflux. No correlation was found with thyroid volume or thyroid pathology.

Conclusions: Swallowing complaints appear to be common in pre-operative thyroidectomy patients. A large percentage, however, have associated laryngopharyngeal reflux which may in part account for these symptoms. Patients presenting with compressive thyroid symptoms should be evaluated for laryngopharyngeal reflux, and treated where appropriate.

Keywords: Surgery, Outcomes, Voice, Swallowing, Thyroid

\section{Introduction}

Compressive symptoms, such as voice, swallowing and airway complaints thought to be secondary to a thyroid pathology, are an accepted indication for thyroidectomy (either subtotal or total thyroidectomy). Treating physicians frequently order thyroid investigations, including thyroid ultrasound, as a part of the work-up when patients present

\footnotetext{
* Correspondence: andersonj@smh.ca

'Department of Otolaryngology - Head \& Neck Surgery, St. Michael's

Hospital, University of Toronto, 30 Bond Street, 8C-129, Toronto M5B 1 W8, ON, Canada

${ }^{2}$ Voice Disorders Clinic, St. Michael's Hospital, University of Toronto, 30 Bond Street, 8 Cardinal Carter North, Toronto M5B 1 W8, ON, Canada
}

with these non-specific symptoms (globus sensation, throat discomfort, swallowing discomfort, voice changes etc.), often revealing the presence of previously undiscovered thyroid nodules. Whether these symptoms are indeed secondary to thyroid pathology or are due to a different process is often unclear. A review of the literature found the reported prevalence rates of voice and swallowing symptoms in pre-operative thyroidectomy patients to vary widely between $11-88 \%$ [1-7].

Although many of these studies report an improvement or resolution of compressive symptoms following thyroidectomy, a number of patients continue to experience

\section{Biomed Central}


voice and swallowing complaints post-operatively [4-8]. Due to the non-specific nature of these complaints, and the possibility of other underlying etiologies, the true prevalence of voice and swallowing symptoms due to thyroid pathology remains unclear. These same symptoms are common in the general population, and are often attributed to and relieved with treatment for laryngopharyngeal reflux (LPR) [9-11]. Currently there is very limited literature examining the prevalence of laryngopharyngeal reflux in thyroidectomy patients. To our knowledge, the only such study was performed by Fiorentino et al. [4], which found that $88 \%$ of patients undergoing thyroidectomy with compressive symptoms had pre-operative evidence of LPR on videofluoroscopic swallow studies (VFSS) [4]. All patients with evidence of LPR on VFSS pre-operatively continued to show changes consistent with LPR on VFSS postoperatively as well. More studies are certainly needed to further examine the prevalence of LPR among preoperative thyroidectomy patients, and to determine its relationship to compressive symptoms.

The purpose of the current study is to determine the overall prevalence and severity of voice and swallowing complaints among preoperative thyroidectomy patients using both subjective and objective measures. In addition, we aim to determine the prevalence of laryngopharyngeal reflux in our cohort of preoperative thyroidectomy patients and to examine its relationship with compressive symptoms. A secondary objective was to determine if any correlation exists between thyroid volume or inflammatory thyroid pathology and the presence and severity of these symptoms.

\section{Methods}

With approval of the Ethics Review Board at our institution, all patients diagnosed with a benign thyroid pathology or well-differentiated thyroid carcinoma (WDTC) who were consented to undergo a thyroidectomy procedure (subtotal or total thyroidectomy) over a 2 year period were invited to participate (October 2008-August 2011). When performed, a partial thyroidectomy consisted of a hemithyroidectomy plus isthmusectomy in all cases. Patients with a history of previous neck surgery (including previous thyroidectomy), prior head and neck radiation, medullary or anaplastic thyroid carcinoma, pre-operative recurrent laryngeal nerve dysfunction or other vocal fold pathology (evaluated using videostroboscopy) were excluded. In order to reduce the risk of age-related voice and swallowing complaints, no patients over the age of 75 years were included in this study. Data on patient factors, such as age, sex, indications for surgery, results of both fine-needle aspiration biopsy and final surgical pathology (including the presence of inflammation or thyroiditis within the specimen) and pre-operative ultrasound characteristics (total thyroid size; number and size of all thyroid nodules) were recorded. Total thyroid gland volume for each patient was calculated from pre-operative ultrasound measurements using the corrective factor 0.479 , as recommended by the World Health Organization [12].

Subjective voice and swallowing complaints were assessed in each patient using the Voice Handicap Index (VHI) [13] and the modified Swallowing Impairment Score (SIS) [5], respectively. Objective acoustic voice measures ((jitter, shimmer, maximum phonation time (MPT), maximum fundamental frequency range (ST range) were collected in a standardized fashion and analyzed using the Multi-Dimensional Voice Program (MDVP) (Kay Elemetrics Version 1.34, 1993). All digital voice recordings were gathered in a sound-treated audiometric booth with each patient seated comfortably in an adjustable chair to maintain constant posture. Acoustic data was recorded with a head mounted microphone (AKG Acoustics, model C410) placed at a 90 degree angle to the left labial angle with the microphone end placed at 45 degrees to the corner of the mouth and out of the air stream $(2 \mathrm{~cm}$ from the lip). Three voice tokens of the sustained vowel /a/ (at habitual pitch and loudness) were recorded for 5 seconds each, and the first 100 milliseconds of voice onset were trimmed from each sample to reduce variability. Each trimmed and isolated vowel token was analyzed for jitter (\%) and shimmer (\%). Maximum phonation time was collected by having the patient sustain the vowel /a/ at habitual pitch and loudness for as long as possible on a single breath following a maximal inhalation. Three trials were obtained, and the best result was retained for further analysis. Maximum fundamental frequency range was calculated in semi-tones and Hertz using the Voice Range Profile program from the Computerized Speech Lab. To calculate the maximum fundamental frequency (F0), the patient was asked to glide from a comfortably low note to their highest note on the vowel $/ \mathrm{u} /$. The best of three trials was recorded. Minimum fundamental frequency was calculated in the same manner was starting at a comfortably high note and gliding to the lowest note on the vowel $/ \mathrm{u} /$. The best of three trials was recorded. Glottal fry was omitted.

Perceptual voice analyses were performed on each patient by a certified Speech Language Pathologist trained in the diagnosis and treatment of voice disorders using the Grade, Roughness, Breathiness, Asthenia, Strain (GRBAS) rating scale [14]. All patients were required to complete the Reflux Symptom Index (RSI) [15] in order to evaluate the presence of concomitant laryngopharyngeal reflux. All data collection was performed in the pre-operative setting, either at the time of enrollment or at the same time as the patients' pre-anaesthetic appointment in order to facilitate ease of data collection and limit additional hospital visits for our patients. 
All statistical analyses were performed using SPSS Statistics Version 20 software. Pearson bivariate correlations using a two-tailed test were performed in order to identify correlations between variables. Jitter, shimmer, MPT and ST Range were compared with published norms using t-tests. Finally, a multivariate analysis of variance (ANOVA) was performed to identify independent relationships among variables. Statistical significance was defined as a $\mathrm{p}$ value less than 0.05 .

\section{Results}

Sixty one patients were invited to participate in this study. Two patients declined enrollment, leaving 59 patients (10 male, 49 female) in total ranging in age from 19-73 years (mean 48.8 years). Twenty four patients (40.7\%) underwent a partial thyroidectomy thyroidectomy, and thirty five (59.3\%) patients had a total thyroidectomy performed. The most common indication for surgery was a finding of atypical cells on fine needle aspiration biopsy (24/59), followed by nodule growth on successive ultrasounds (8/59). Six patients underwent a thyroidectomy procedure for the sole indication of compressive symptoms (Table 1). Thirty three patients

\section{Table 1 Patient demographics}

\begin{tabular}{ll}
\hline Sex: & 10 \\
Male & 49 \\
Female & $\mathbf{4 8 . 6}$ years (19-73) \\
Age: & \\
Surgery: & $\mathbf{2 4}$ \\
$\quad$ Partial Thyroidectomy & $\mathbf{3 5}$ \\
Total thyroidectomy & \\
Pathology: & 33 \\
Benign & 16 \\
$\quad$-Multinodular Goitre & 8 \\
$\quad$-Hyperplastic nodule & 7 \\
$\quad$-Follicular/hurthle cell adenoma & 2 \\
$\quad$-Graves disease & $\mathbf{3 6}$ \\
Malignant (WDTC) & \\
Indications for surgery: & $\mathbf{2 6}$ \\
Compressive symptoms & $\mathbf{6}$ \\
Multinodular goiter & $\mathbf{7}$ \\
Atypical cells & $\mathbf{2 1}$ \\
Follicular/Hurthle cell lesion & $\mathbf{4}$ \\
Nodule growth & $\mathbf{8}$ \\
Graves disease & \\
Suspected WDTC & $\mathbf{3}$ \\
Known WDTC & \\
\hline
\end{tabular}

(55.9\%) were diagnosed with benign thyroid pathology, and 26 patients $(44.1 \%)$ with well-differentiated thyroid carcinoma. Of the patients with a diagnosis of WDTC, 9 were reported as microscopic papillary thyroid carcinoma. The average total thyroid volume on ultrasound was $38.7 \mathrm{~cm}^{3}$ (range $6.3-237.9 \mathrm{~cm}^{3}$ ). There were no retrosternal thyroid goitres in this patient cohort. Patient characteristics, indications for surgery and final thyroid pathology are summarized in Table 1.

Nineteen patients (32.2\%) reported scores consistent with moderate to severe voice complaints on the VHI (9/59 moderate, 10/59 severe). All of the voice complaints were within the functional and physical domains, with no patients reporting elevated scores in the emotional domain. The average VHI score was 10.8, however, falling within the normal range. Objective acoustic voice analysis found jitter to be significantly elevated compared with normative data at $1.28(\mathrm{p}=0.03)$; while shimmer was not significantly elevated. MPT and ST range were both within accepted norms (Table 2). Similarly, perceptual voice analysis using the GRBAS scoring system by a trained speech language pathologist did not reveal any significant abnormalities (G-0.52, R-0.62, B-0.33, A-0.16, S-0.35; p > 0.5).

Twenty five patients $(42.7 \%)$ reported swallowing symptoms at least some of the time on the MSIS, with 16 patients $(27.1 \%)$ reporting symptoms often or always. The average score on the MSIS was 6.5 out of a possible total score of 28 , with the majority of symptoms relating to a globus sensation (Q5-7: "I always feel a sensation of lump in my throat", "I feel a sensation of a lump in my throat when I lay down", "I feel a sensation of a lump in my throat when I move my head").

Twenty patients (33.9\%) scored positive on the RSI for laryngopharyngeal reflux. Of the 19 patients with an elevated VHI, 68.4\% (13/19) had an elevated RSI score consistent with LPR. Similarly, 56\% (14/25) of patients with swallowing complaints also had scores on the RSI suggestive of laryngopharyngeal reflux. In addition, a positive correlation was found between MSIS and RSI scores $(\mathrm{p}<0.001)$ indicating that those who scored highest with regards to swallowing complaints also scored highest for LPR.

When compared with thyroid volume on ultrasound, there was no significant correlation between VHI, jitter, shimmer, MPT, MSIS or RSI. There did appear to be a

Table 2 Acoustic voice outcomes

\begin{tabular}{llll}
\hline Variable & $\begin{array}{l}\text { Pre-op thyroidectomy } \\
\text { (mean) }\end{array}$ & $\begin{array}{l}\text { Normative } \\
\text { value (mean) }\end{array}$ & P-value \\
\hline Jitter (\%) & 1.28 & $\leq 1.04$ & $\mathbf{0 . 0 3}^{*}$ \\
Shimmer (\%) & 3.83 & $\leq 3.81$ & $>0.05$ \\
MPT (seconds) & 18.80 & $\geq 15$ & $>0.05$ \\
ST Range (dB) & 30.29 & $\geq 27$ & $>0.05$ \\
\hline
\end{tabular}


negative correlation between ST range and thyroid volume. That is, as thyroid volume increases, the number of semitones a person has in their range decreases $(p=0.045)$. No significant relationships were found between the presence of inflammatory thyroid pathology and any of the above measures (Table 3).

\section{Discussion}

The prevalence of self-reported voice and swallowing complaints in our cohort was found to be $32.2 \%$ and $42.7 \%$, respectively. A literature review found that the reported prevalence of compressive symptoms in pre-operative thyroidectomy patients varied widely between studies, ranging from 11-88\% [1-7]. Unfortunately many of these studies vary with regards to the symptoms reported as well as the methods used for data collection (e.g. patient interviews, visual analogue scales, subjective patient questionnaires), making comparisons between cohorts difficult.

Despite $32.2 \%$ of patients reporting moderate to severe voice complaints on the VHI, the overall mean score of 10.8 was not significantly elevated (although trending toward moderate dysfunction). Objective acoustic voice analysis showed only jitter to be significantly elevated in our cohort at $1.28 \%$. In addition, perceptual analysis by a trained Speech Language Pathologist using the GRBAS scoring system did not reveal significant abnormalities in voice quality, despite patient perceptions of dysfunction. This is in agreement with the results from several studies where jitter, shimmer, MPT, ST range, and GRBAS measures were all within the normal range; often despite patient complaints of voice abnormalities [5,6,16,17]. In addition, studies have shown that $14-75 \%$ of patients continue to complain of voice symptoms 3 months -1 year post thyroidectomy, questioning the origin of these symptoms with regards to thyroid disease $[4-6,16]$.

Swallowing dysfunction is a commonly reported symptom in pre-operative thyroidectomy patients, with the majority complaining of a globus sensation [1,3-5,7,18]. In our cohort, $47.2 \%$ of patients reported swallowing

Table 3 Effect of thyroid volume and inflammatory pathology

\begin{tabular}{lll}
\hline & $\begin{array}{l}\text { U/S thyroid } \\
\text { volume }(\boldsymbol{p} \text { value) }\end{array}$ & $\begin{array}{l}\text { Inflammatory } \\
\text { pathology ( } \mathbf{p} \text { value) }\end{array}$ \\
\hline VHI & $>0.05$ & $>0.05$ \\
Jitter & $>0.05$ & $>0.05$ \\
Shimmer & $>0.05$ & $>0.05$ \\
MPT & $>0.05$ & $>0.05$ \\
Semitone Range & $>0.045^{*}$ & $>0.05$ \\
MSIS & $>0.05$ & $>0.05$ \\
RSI & $>0.05$ & $>0.05$ \\
\hline
\end{tabular}

*Negative correlation. symptoms at least some of the time, with $27.1 \%$ reporting symptoms often or always; with the majority of symptoms relating to a globus sensation. Although many studies do show improvement in swallowing complaints post-thyroidectomy, a variable proportion of patients continue to report symptoms 3 months to 1 year post-thyroidectomy (20-91\%) [3-5,18].

Given the fact that there does appear to be a subgroup of pre-operative thyroidectomy patients with voice and swallowing complaints that does not respond to thyroidectomy, we questioned the possible co-existence of laryngopharyngeal reflux in these patients. As such, we administered the RSI to our patients and found that 33.9\% of pre-operative thyroidectomy patients had concomitant laryngopharyngeal reflux, which is in keeping with reported LPR rates in the general population [9-11]. However, when we looked specifically at the relative contribution of LPR to voice and swallowing complaints in this cohort we found that laryngopharyngeal reflux may account for a larger proportion of patients with elevated symptom scores. That is, $68.4 \%$ of patients with elevated VHI scores, as well as 56\% of patients with swallowing complaints on the MSIS also scored positive for LPR. To our knowledge, the only other study to date to look at LPR in thyroidectomy patients is Fiorentino et al. [4], who found that $88 \%$ of pre-operative thyroidectomy patients with compressive symptoms also had pre-operative evidence of LPR on videofluoroscopic swallow studies (VFSS) [4]. In addition, all patients with evidence of LPR on VFSS pre-operatively continued to show changes consistent with LPR on VFSS post-operatively as well. Further studies are needed in order to explore the relationship between compressive symptoms and LPR, as well as to determine the impact of reflux treatment on postthyroidectomy symptomatology.

\section{Conclusions}

Compressive symptoms, such as voice and swallowing complaints, are an accepted indication for thyroidectomy (either total or partial). This study shows the prevalence of voice and swallowing complaints in pre-operative thyroidectomy patients to be $32.2 \%$ and $42.7 \%$, respectively. In addition, we show that a large percentage of patients presenting with voice and swallowing complaints may have associated laryngopharyngeal reflux, which can at least in part account for these symptoms. As such, all patients presenting with compressive thyroid symptoms should also be evaluated for laryngopharyngeal reflux, and treated where appropriate prior to performing a thyroidectomy.

Competing interests

The authors declare they have no competing interests. 


\section{Authors' contributions}

TH was involved in collection and analysis of data, as well as drafting the manuscript. JA was involved in collection and analysis of data, as well as manuscript preparation and editing. Both authors read and approved the final manuscript.

\section{Acknowledgements}

Merrick G MHSc Reg. CASLPO, DeLuca M MCISc Reg. CASLPO,

McNelles L PhD.

Presented at the Canadian Society of Otolaryngology - Head \& Neck Surgery 66 Annual Meeting, Toronto, ON, Canada (May 22, 2012)

Received: 9 September 2013 Accepted: 5 July 2014

Published: 15 July 2014

\section{References}

1. Banks CA, Ayers CM, Hornig JD, Lentsch MD, Day TA, Nguyen SA, Gillespie MB: Thyroid disease and compressive symptoms. Laryngoscope 2011, 122:13-16.

2. Lacoste L, Gineste D, Karayan J, Montaz N, Lehuede MS, Girault M, Bernit AF, Barbier J, Fusciardi J: Airway complications in thyroid surgery. Ann Otol Rhinol Laryngol 1993, 102:441-446.

3. Burns $P$, Timon C: Thyroid pathology and the globus symptom: are they related? A two year prospective trial. J Laryngol Otol 2007, 121:242-245.

4. Fiorentino E, Cipolla C, Graceffa G, Cusimano A, Cupido F, Re GL, Matranga D, Latteri MA: Local neck symptoms before and after thyroidectomy: a possible correlation with reflux laryngopharyngitis. Eur Arch Otorhinolaryngo/ 2011 , 268:715-720.

5. Lombardi CP, Raffaelli M, De Crea C, D'Alatri L, Maccora D, Marchese MR, Paludetti G, Bellantone R: Long-term outcome of functional post-thyroidectomy voice and swallowing symptoms. Surgery 2009 , 146:1174-1181.

6. Van Lierde K, D'haeseleer E, Wuyts FL, Baudonck N, Bernaert L, Vermeersch H: Impact of Thyroidectomy Without Laryngeal Nerve Injury on Vocal Quality Characteristics: An Objective Multiparameter Approach. Laryngoscope 2009, 120:338-345

7. Greenblatt DY, Sippel R, Leverson G, Frydman J, Schaefer S, Chen H: Thyroid Resection Improves Perception of Swallowing Function in Patients with Thyroid Disease. World J Surg 2009, 33:255-260.

8. Pereira JA, Girvent M, Sancho JJ, Parada C, Sitges-Sierra A: Prevalence of long-term upper aero-digestive symptoms after uncomplicated bilateral thyroidectomy. Surgery 2003, 133:318-322.

9. Selby JC, Gilbert HR, Lerman JW: Perceptual and acoustic evaluation of individuals with laryngopharyngeal reflux pre- and post-treatment. J Voice 2003, 17(4):557-570.

10. Lee YS, Choi SH, Son YI, Park YK, Kim SY, Nam SY: Prospective, observational study using rabeprazole in 455 patients with laryngopharyngeal reflux disease. Eur Arch Otorhinolaryngol 2011, 268(6):863-869.

11. Koufman JA, Aviv JE, Casiano RR, Shaw GY: Laryngopharyngeal reflux: position statement of the committee on speech, voice and swallowing disorders of the American Academy of Otolaryngology-Head and Neck Surgery. Otolaryngol Head Neck Surg 2002, 127(1):32-35.

12. Karachenko VP, Kotlyarov PM, Mogutov MS, Alexandrov YK, Sencha AN, Patrunov YN: Ultrasound Diagnostics of Thyroid Diseases. 1st edition. Berlin Heidelberg: Springer-Verlag; 2010.

13. Jacobson BH, Johnson A, Grywalski C, Silbergleit A, Jacobson G, Benninger MS, Newman CW: The Voice Handicap Index (VHI): Development and Validation. Am J Speech Lang Pathol 1997, 6(3):66-70.

14. Hirano M: Clinical Examination of Voice. New York: Springer Verlag 1981:81-84.

15. Belafsky PC, Postma GN, Koufman JA: Validity and reliability of the reflux symptom index (RSI). J Voice 2002, 16(2):274-277.

16. Stojadinovic A, Shaha AR, Orlikoff RF, Nissan A, Kornak M, Singh B, Boyle JO, Shah JP, Brennan MF, Kraus D: Prospective functional voice assessment in patients undergoing thyroid surgery. Ann Surg 2002, 236(6):823-832.
17. Soylu L, Ozbas S, Uslu HY, Kocak S: The evaluation of the causes of subjective voice disturbances after thyroid surgery. Am J Surg 2007, 194:317-322.

18. Maung KH, Hayworth D, Nix PA, Atkin SL, England RJA: Thyroidectomy does not cause globus pattern symptoms. J Laryngol Otol 2005, 119(12):973-975.

doi:10.1186/s40463-014-0028-4

Cite this article as: Holler and Anderson: Prevalence of voice \& swallowing complaints in Pre-operative thyroidectomy patients: a prospective cohort study. Journal of Otolaryngology - Head and Neck Surgen 2014 43:28.

\section{Submit your next manuscript to BioMed Central and take full advantage of:}

- Convenient online submission

- Thorough peer review

- No space constraints or color figure charges

- Immediate publication on acceptance

- Inclusion in PubMed, CAS, Scopus and Google Scholar

- Research which is freely available for redistribution

Submit your manuscript at www.biomedcentral.com/submit
C Biomed Central 